\title{
Nickel(II) tetra-aminophthalocyanine modified MWCNTs as potential nanocomposite materials for the development of supercapacitors $\dagger$
}

\author{
Alfred T. Chidembo, ${ }^{a}$ Kenneth I. Ozoemena, ${ }^{* a b}$ Bolade O. Agboola, ${ }^{b}$ Vinay Gupta, ${ }^{c}$ Gregory G. Wildgoose $\uparrow^{d}$ \\ and Richard G. Compton ${ }^{d}$
}

Received 4th August 2009, Accepted 8th October 2009

First published as an Advance Article on the web 12th November 2009

DOI: $10.1039 / \mathrm{b} 915920 \mathrm{~g}$

\begin{abstract}
The supercapacitive properties of nickel(II) tetraaminophthalocyanine (NiTAPc)/multi-walled carbon nanotube (MWCNT) nanocomposite films have been interrogated for the first time and found to possess a maximum specific capacitance of $981 \pm 57 \mathrm{~F} \mathrm{~g}^{-1}\left(200 \pm 12 \mathrm{mF} \mathrm{cm}^{-2}\right)$, a maximum power density of $700 \pm 1 \mathrm{Wkg}^{-1}$, a maximum specific energy of $134 \pm 8 \mathrm{Wh} \mathrm{kg}^{-1}$ and excellent stability of over 1500 charge-discharge continuous cycling. Impedimetric study proves that most of the stored energy of the MWCNT-NiTAPc nanocomposite can be accessible at high frequency $(720 \mathrm{~Hz})$. When compared to MWCNTs modified with unsubstituted nickel(II) phthalocyanine (MWCNT-NiPc) or nickel(II) tetratert-butylphthalocyanine (MWCNT- $t$ BuNiPc), MWCNT-NiTAPc exhibited superior supercapacitive behaviour, possibly due to the influence of nitrogen-containing groups on the phthalocyanine rings.
\end{abstract}

\section{Introduction}

Electrochemical capacitors (also known as "supercapacitors" or "ultracapacitors" or "power capacitors") are charge-storage devices with a high power density and long cycle life. They serve as intermediate systems that bridge the power/energy gap

${ }^{a}$ Department of Chemistry, University of Pretoria, Pretoria, 0002, South Africa

${ }^{b}$ Energy and Processes Unit, Materials Science and Manufacturing, Council for Scientific and Industrial Research (CSIR), Pretoria, 0001, South Africa. E-mail: kozoemena@csir.co.za; Fax: +27(0)12 841 2135; Tel: $+27(0) 128413664$

'Carbon Technology Unit, National Physical Laboratory, New Delhi, 110012, India

${ }^{d}$ Physical and Theoretical Chemistry Laboratory, University of Oxford, South Parks Road, Oxford, UK

$\dagger$ Electronic supplementary information (ESI) available: Molecular structures; charge-discharge curves; cyclic voltammetry data. See DOI: $10.1039 / \mathrm{b} 915920 \mathrm{~g}$

† Present address: School of Chemistry, University of East Anglia, Norwich, UK between traditional dielectric capacitors (high power) and batteries (high energy). ${ }^{1}$ This is an active area of research seeking to develop the next generation of cost-effective, energy-efficient and environmentally-friendly power sources for a variety of everyday applications. ${ }^{1,2}$

There are two types of supercapacitors, viz: (a) "electrochemical double layer capacitor" (EDLC) and (b) "pseudocapacitors". The operation mechanism of the former involves the non-Faradaic separation of charges at the "double-layer" (i.e., electrode/electrolyte interface) while the latter involves fast Faradaic, redox reaction of electroactive materials at the interface. ${ }^{3}$ EDLCs are obtained from carbon materials such as activated carbons, carbon aerogels and carbon nanotubes, while pseudocapacitors are obtained from redox-active materials such as polymeric complexes and metal oxides. Hybrid materials incoporating EDLC and pseudocapacitor materials are thought to give the next generation high-performance supercapacitor devices.

Metallophthalocyanine (MPc) complexes are metal N4 macrocyclic organometallic complexes known for their excellent

\section{Broader context}

Electrochemical capacitors (also known as supercapacitors) represent an important electrochemical energy storage and delivery system. When compared to batteries, supercapacitors give more power density. Presently, this is an active area of research seeking to develop the next generation of cost-effective, energy-efficient and environmentally-friendly power sources for a variety of applications (such as in potable electronics and hybrid electric vehicles). The nickel tetraaminophthalocyanine (NiTAPc) complex is a member of the metallophthalocyanine family, well known for their excellent physico-chemical and redox properties. Although MPc complexes have been known to show interesting properties for potential applications in a plethora of areas such as in catalysis, sensing and photocatalysis, until now there has not been any report on their possible application for the development of supercapacitors. In this work, we have shown for the first time that multi-walled carbon nanotubes (MWCNTs) and NiTAPc nanocomposites could potentially be used as electrode materials for the development of high-performance supercapacitors. Given the high number of existing MPc complexes and new ones constantly reported in the literature, and the importance of supercapacitors in energy development, we envisage that this novel study is likely to spark research interests in the supercapacitive properties of CNTMPc hybrid electrodes. 
physico-chemical and redox properties. ${ }^{4-7}$ Although MPc complexes have been known to show interesting properties for potential applications in a plethora of areas such as in catalysis, sensing and photocatalysis, until now, there has been no report on their supercapacitive behaviour. A recent patent on EDLC based on activated carbon claimed the use of unsubstituted FePc and $\mathrm{CoPc}$ as mere 'conductive agents', ${ }^{8}$ it however did not give any details on either the electrochemistry or carried out any study on the impedimetric property of the electrodes. This work interrogates for the first time the supercapacitive behaviour of a model MPc complex, nickel(II) tetraaminophthalocyanine (NiTAPc, Fig. 1) integrated into a high surface area conducting carbon nanotubes (CNTs) support.

Our choice of amino-substituted NiPc complex supported on MWCNTs has been motivated by many factors such as: (i) costeffectiveness and ease of preparation of MPc complexes, notably NiTAPc; (ii) the high popularity of $\mathrm{Ni}$ complexes as supercapacitive materials ${ }^{9}$ compared to $\mathrm{Fe}$ or Co complexes; (iii) the enhanced supercapacitive properties of nitrogen-containing materials; ${ }^{10-12}$ and (iv) the ability to be stably immobilized onto a basal plane pyrolytic graphite substrate via $\pi-\pi$ interaction; (v) the high insolubility of NiTAPc in aqueous solution, an essential property that prevents it to from being washed away from its carbon electrode substrate when used in an aqueous system; and (vi) the high surface area, high conductivity and interconnectivity of CNTs that enhance capacitive properties. ${ }^{13}$

In this work, we show for the first time, using cyclic voltammetry (CV), galvanostatic charge/discharge (CD) and electrochemical impedance spectroscopy (EIS) techniques, that an MWCNT-NiTAPc nanocomposite film immobilized onto a basal plane pyrolytic graphite electrode (BPPGE) is a promising platform for potential development of supercapacitors in an aqueous, acidic electrolyte.

\section{Experimental details}

\subsection{Materials and reagents}

Multi-walled carbon nanotubes (MWCNT, purity $>90 \%$, $110-170 \mathrm{~nm}$ in diameter, 5-9 $\mu \mathrm{m}$ in length) obtained from Sigma Aldrich were acid-functionalised by undergoing stringent acid treatment following the established methods. ${ }^{14-17}$ The MWCNTs were purified as described previously. Briefly, $1 \mathrm{~g}$ of MWCNTs in

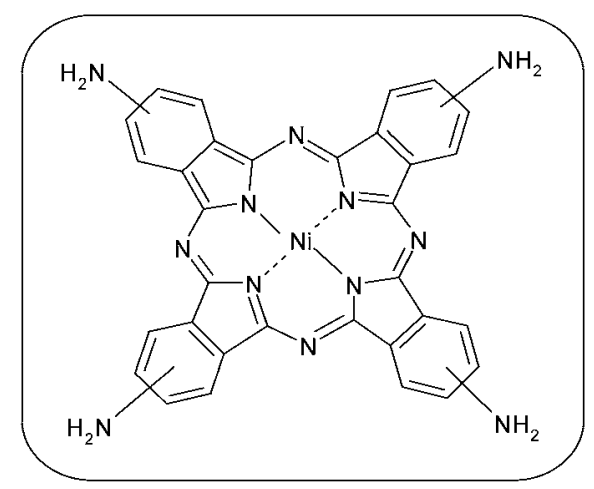

Fig. 1 Molecular structure of the nickel(II) tetra-amino phthalocyanine (NiTAPc) complex.
$140 \mathrm{ml}$ of $2.6 \mathrm{M} \mathrm{HNO}_{3}$ was refluxed for $48 \mathrm{~h}$. The carbon nanotube sediments were separated from the reaction mixture, washed with distilled water, and sonicated in a concentrated $\mathrm{H}_{2} \mathrm{SO}_{4}-\mathrm{HNO}_{3}$ mixture $(3: 1$ ratio, $98 \%$ and $70 \%$ purity, respectively) for $24 \mathrm{~h}$. Thereafter, the sediments were washed with distilled water, stirred for 30 min in a $\mathrm{H}_{2} \mathrm{SO}_{4}-\mathrm{H}_{2} \mathrm{O}_{2}$ mixture (4:1, 98\% and $30 \%$ purity, respectively) at $70{ }^{\circ} \mathrm{C}$, and washed again with distilled water. Finally, the purified paste-like MWCNTs were oven-dried at $50{ }^{\circ} \mathrm{C}$ for $48 \mathrm{~h}$.

Nickel(II) tetraaminophthalocyanine (NiTAPc, Fig. 1) was synthesised and characterised following the well-known procedure introduced by Acher and Jayasree. ${ }^{18}$ For comparative studies, unsubstituted nickel phthalocyanine (NiPc, Aldrich) and nickel(II) tetra-tert-butyl phthalocyanine $(t \mathrm{BuNiPc}$, Fig. 1 in the ESI $\dagger$ ) were investigated for their supercapacitive behaviour. The $t \mathrm{BuNiPc}$ was obtained from its metal-free precursor, tetra-tertbutyl phthalocyanine ( $t \mathrm{BuPc}$, kindly donated by Prof T. Fukuda, Graduate School of Science, Tohoku University, Japan). Introduction of nickel into the core of the $t \mathrm{BuPc}$ was achieved using a similar metallation process described before ${ }^{19}$ Briefly, this involved refluxing a DMF solution containing a mixture of $t \mathrm{BuPc}$ and an excess of nickel acetate for about an hour. The DMF was then evaporated and the dark green residue purified using a short silica gel column $\left(\mathrm{CHCl}_{3}\right.$ with a small amount of methanol as eluent) to afford the desired $t$ BuNiPc. As expected, the formation of the nickel complex was easily established by UV-visible spectrophotometry in $\mathrm{CHCl}_{3}$ by observing the total collapse of the split Q-bands (663 and $696 \mathrm{~nm}$ ) of the metal-free $t \mathrm{BuPc}$ complex ( $D_{2 \mathrm{~h}}$ symmetry) to the single Q-band $(668 \mathrm{~nm})$ of the metal, $t \mathrm{BuNiPc}$, complex ( $D_{4 \mathrm{~h}}$ symmetry). Ultra-pure water of resistivity $18.2 \mathrm{M} \Omega \mathrm{cm}$ was obtained from a Milli-Q Water System (Millipore Corp., Bedford, MA, USA) and was used throughout the experiments.

\subsection{Preparation of NiTAPc-MWCNT composite and electrode fabrication}

Prior to modification with nickel phthalocyanine complexes or MWCNTs or their MWCNT composites, the carbon electrode substrate (BPPGE surface) was cleaned by gentle polishing on a p1200C Norton carborundum paper (Saint-Goban Abrasives, Isando, South Africa) followed by cleaning with the cellotape process of removing graphite layers and finally rinsing in acetone to remove any adhesives. The MWCNT-NiTAPc composite was obtained by grinding an equal amount of MWCNT and NiTAPc (1:1 mass/mass ratio), dissolved in DMF, ultrasonicated for 30 min and finally dried. Unlike the pristine MWCNTs, the acidfunctionalised MWCNTs exhibited an excellent dispersion in dry distilled DMF. NiTAPc, acid-treated MWCNTs and MWCNTNiTAPc are highly dispersible in dry DMF. Neither of them in DMF showed any detectable precipitate even after 3 months. However, the dispersion/solutions were used within $48 \mathrm{~h}$ of preparation. The drop-dry strategy was employed to load the NiTAPc or MWCNTs or MWCNT-NiTAPc hybrids onto the BPPGE surface by placing the required drops $(20-80 \mu \mathrm{L})$ of the dispersion/solution material (1 mg material dissolved in $1 \mathrm{~mL}$ dry DMF) onto the BPPGE surface and dried in the oven at $50{ }^{\circ} \mathrm{C}$ for about $2 \mathrm{~min}$. 


\subsection{Equipments and methods}

Field emission scanning electron microscopy (FESEM) images were obtained from JEOL JSM 5800 LV (Japan). Atomic force microscopy (AFM) experiments were performed with AFM 5100 System (Agilent Technologies, USA). TGA was performed with a Mettler Toledo A851 (Switzerland). A simultaneous TGA/ DTA machine was used for thermal and gravimetric analysis. Powder samples ( $c a .10 \mathrm{mg}$ ) were heated from $25-1000{ }^{\circ} \mathrm{C}$ at a scan rate of $10{ }^{\circ} \mathrm{C} \mathrm{min}^{-1}$ in nitrogen atmosphere. Infra-red spectra were obtained with the Perkin-Elmer GX 2000 FTIR spectrometer attached to the Perkin-Elmer Auto Image Microscope system equipped with a liquid Nitrogen-cooled MCT detector. Mass measurements, unless otherwise stated, were performed with the Sartorius CP225D micro-balance with a readability $(d)$ of $0.01 \mathrm{mg}$.

Electrochemical experiments were carried out using an Autolab Potentiostat PGSTAT 100 (Eco Chemie, Utrecht, Netherlands) driven by the GPES software version 4.9. Electrochemical impedance spectroscopy (EIS) measurements were performed with Autolab Frequency Response Analyser (FRA) software between $100 \mathrm{kHz}$ and $10 \mathrm{mHz}$ using a $5 \mathrm{mV}$ rms sinusoidal modulation. A bare or modified BPPGE disk $(d=5 \mathrm{~mm}$ in Teflon) was used as the working electrode and was fabricated from BPPG plate (Le Carbone, Sussex, UK). Electrical contact with the disk was maintained through an inserted copper wire held in place with conducting silver varnish. $\mathrm{An} \mathrm{Ag} \mid \mathrm{AgCl}$, saturated $\mathrm{KCl}$ and platinum wire were used as reference and counter electrodes, respectively. All solutions were saturated with nitrogen prior to each electrochemical experiment. Galvanostatic charge/discharge (CD) experiments were carried out at different current densities of 1-30 $\mathrm{A} \mathrm{g}^{-1}$ (i.e., $0.1-3 \mathrm{~mA} \mathrm{~cm}^{-2}$ ) in $1 \mathrm{M}$ $\mathrm{H}_{2} \mathrm{SO}_{4}$. Experiments were performed at $25 \pm 1{ }^{\circ} \mathrm{C}$.

\section{Results and discusion}

\subsection{FESEM and XRD charaterisation}

Fig. 2 shows the FESEM images of the MWCNT-NiTAPc nanocomposite. Fig. 2 (inset) clearly reveals the attachment (to a certain extent, via $\pi-\pi$ interactions) of the NiTAPc nanoaggregates (50-100 $\mathrm{nm}$ size) on the nanofibril-like structure of the acid-functionalised MWCNTs. The FESEM image of the

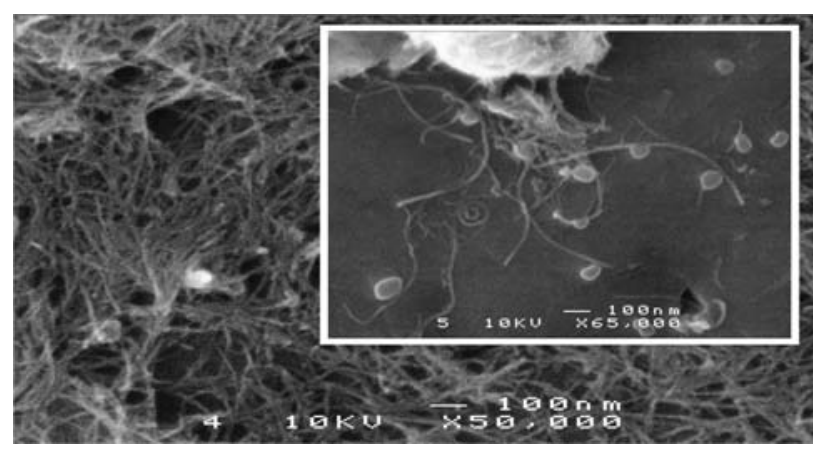

Fig. 2 Typical FESEM image of MWCNT-NiTAPc nanofibril composites $(\times 50000)$. Inset exemplifies a slightly magnified portion ( $\times 65000)$ showing some of the NiTAPc nano-aggregates $(c a .50-100 \mathrm{~nm})$ attaching themselves onto the cylindrical structure of the MWCNTs.
MWCNT-NiTAPc is different from that reported by Wang et $a l^{20}$ of a metal-free phthalocyanine (tetra-tert-butyl phthalocyanine, $t \mathrm{BuPcs}$ ) species that were uniformly and non-covalently attached to the CNTs via $\pi-\pi$ interactions. The authors ${ }^{20}$ noted that when the $t$ BuPcs was metallated with copper to form the copper(II) tetra-tert-butyl phthalocyanine ( $t$ BuCuPcs), these $\pi-\pi$ interactions were hindered, which they attributed to the destruction of the aromaticity of the conjugated $t \mathrm{BuCuPcs}$ backbone due to the formation of the coordinate bond between copper and the nitrogens of the core $t$ BuPcs. This explanation may partly hold for our MWCNT-NiTAPc, but their noncovalent attachment cannot be ruled out, as other workers ${ }^{21}$ have also proved this to be possible with nanocomposites of SWCNTs/metallated porphyrins (compounds structurally related to phthalocyanines).

The XRD patterns of the MWCNTs and NiTAPc powders are essentially the same but differ slightly from that of the MWCNTNiTAPc hybrid (Fig. 3a), confirming the formation of a new material and a possible change in crystallinity and interplanar space. ${ }^{15}$ TGA curve of the MWCNTs (Fig. 3b(i)) shows a steady loss of weight from about $100{ }^{\circ} \mathrm{C}$ up to about $20 \%$ at the end of the pyrolysis at $1000^{\circ} \mathrm{C}$. This is interesting considering that the TGA of CNTs, perfomed under similar conditions as ours, showed a low amount of weight loss $(<10 \%)$ when pyrolysed to about $700{ }^{\circ} \mathrm{C}$. This discrepancy may be due to the harsh treatment adopted in the functionalisation of our MWCNTs that introduced oxo-groups on their surfaces. The NiTAPc (ii) follows the same pattern as the MWCNTs except that it showed drastic reduction of weight from about $540{ }^{\circ} \mathrm{C}$, just like the MWCNT-NiTAPc (iii). TGA curve of the MWCNT-NiTAPc (iii) shows an initial weight loss of about $15 \%$ starting from $100{ }^{\circ} \mathrm{C}$ until around $200{ }^{\circ} \mathrm{C}$ due to the desorption of physically adsorbed water. The onset pyrolysis of the MWCNT-NiTAPc was at about $200{ }^{\circ} \mathrm{C}$. Further decomposition of the MWCNTNiTAPc complex started at about $540{ }^{\circ} \mathrm{C}$ until $1000{ }^{\circ} \mathrm{C}$.

The thermal stability decreases as MWCNT $>$ NiTAPc $>$ MWCNT-NiTAPc. Wang et al. ${ }^{20}$ also observed similar trend wherein the onset pyrolysis temperature of the $t \mathrm{BuPcs} / \mathrm{CNTs}$ $\left(213{ }^{\circ} \mathrm{C}\right)$ was remarkably lower than that of the $t$ BuPcs $\left(465.8{ }^{\circ} \mathrm{C}\right)$. The authors ${ }^{20}$ attributed the low decompsition temperature of the $t \mathrm{BuPcs} / \mathrm{CNT}$ s to the increased internal energy
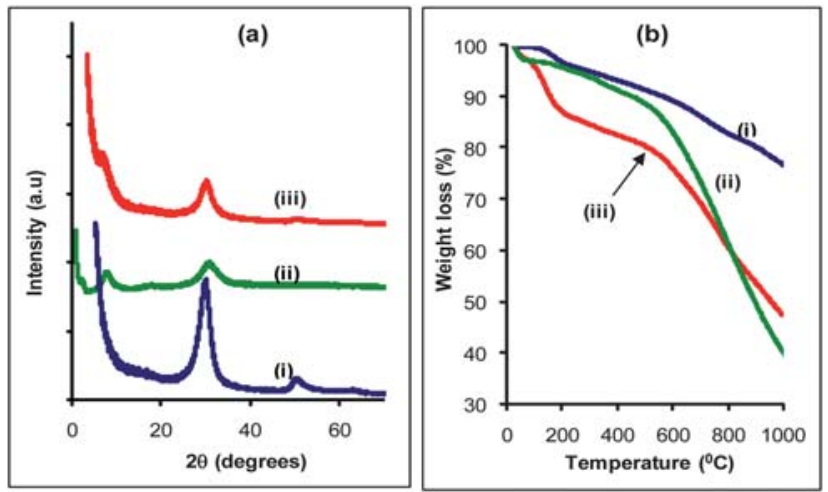

Fig. 3 Comparative XRD (a) and TGA (b) of acid-functionalised MWCNTs (i), bulk NiTAPc (ii) and MWCNT-NiTAPc nanocomposite (iii). 
of the adsorbed $t$ BuPcs. The same explanation may also explain the trend we observed in our results. In other words, the lower thermal stability of the MWCNT-NiTAPc compared to the MWCNTs or NiTAPc may be due to the increased internal energy of the bulk NiTAPc that results from structural configuration following the attachment of NiTAPc nano-aggregates onto the nanofibril structure of the MWCNTs.

\subsection{Cyclic voltammetric characterisation}

Fig. $4 \mathrm{a}$ is the overlaid cyclic voltammograms of the (i) BPPGE, (ii) BPPGE-NiTAPc, (iii) BPPGE-MWCNT and (iv) BPPGEMWCNT-NiTAPc in $1 \mathrm{M} \mathrm{H}_{2} \mathrm{SO}_{4}$ solution, which clearly shows that the NiTAPc/MWCNT nanocomposite exhibits a larger capacitance compared to the other electrodes. For clarity purposes, Fig. 4b presents only the cyclic voltammograms of the (i) BPPGE (ii) BPPGE-NiTAPc and (iii) BPPGE-MWCNT. It is evident from Fig. 4b that the acid-functionalised MWCNTs are associated with surface reactions with peaks at $c a$. $-0.1,0.05$, $0.2 \mathrm{~V}$ instead of the typical rectangular shape expected of an EDLC behaviour of carbon materials as MWCNTs. This behaviour is in good agreement with literature and should certainly be expected considering the long digestion period employed in the functionalisation of these MWCNTs. The specific capacitance for each modifier is estimated using the reliable galvanostatic charge/discharge method (see section 3.3).

In acidic medium, pseudocapacitance arises from the redox reactions of the functional groups on the surface of the materials. In the case of acid pretreated MWCNTs these are likely to correspond to the two-electron, two-proton redox process of surface quinones, which are also introduced as surface oxogroups during acid pretreatment of MWCNTs. ${ }^{22-24}$ These surface quinones give rise to two small, ill-defined, quasireversible redox waves observed at $c a$. $0.2 \mathrm{~V} v$ s. SCE at low $\mathrm{pH}$, corresponding to both ortho- and para-quinone surface groups. In fact, a closer look at Fig. 4 shows that those peaks observed for the MWCNTs (Fig. 4b) are also present (as weak peaks) at the MWCNTNiTAPc voltammogram (Fig. 4a). Thus, the broad redox couple (indicative of pseudo-capacitance) of the MWCNT-NiTAPc nanocomposite (Fig. 4a) at around $0.2 \mathrm{~V}$ is related to both the quinonyl oxygen functionalities of the acid-functionalised
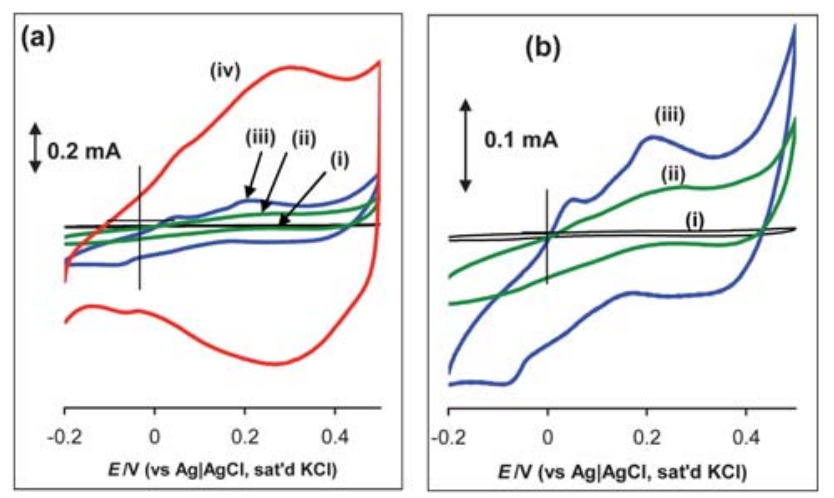

Fig. 4 (a) Overlaid cyclic voltammograms comparing a bare BPPGE (i), BPPGE-NiTAPc (ii), BPPGE-MWCNT (iii) and (BPPGE-MWCNTNiTAPc (iv) at $0.05 \mathrm{~V} \mathrm{~s}^{-1}$. (b) Expansion of the voltammograms of the (i) to (iii). Electrolyte $=1 \mathrm{M} \mathrm{H}_{2} \mathrm{SO}_{4}$.
MWCNTs as well as the $\mathrm{Ni}(\mathrm{II}) / \mathrm{Ni}(\mathrm{III})$ redox process of the NiTAPc species (eqn (1)):

$$
\mathrm{Ni(II)TAPc} \rightleftharpoons \mathrm{Ni(III)TAPc}+\mathrm{e}^{-}
$$

The participation of the nitrogen-containing groups of the NiTAPc in the electrochemistry may not be completely ruled out. For example, For example, redox chemistry corresponding to imine-like groups within the phthalocyanine structure, which is analogous to the quinonyl redox processes discussed above, may also be a contributory factor to the pseudo-capacitance observed in Fig. 4.

$$
\mathrm{C}^{*}=\mathrm{NH}+2 \mathrm{e}^{-}+2 \mathrm{H}^{+} \rightleftharpoons \mathrm{C}^{*} \mathrm{H}-\mathrm{NH}_{2}
$$

According to Hulicova-Jurcakova et al. ${ }^{12}$ pyrrol and pyridinic nitrogens are electroactive and can enhance supercapacitance. This is because pyrrol or pyrrol-like nitrogens $(-\mathrm{NH})$ improve charge mobility in a carbon matrix by their ability to introduce electron-donor characteristics and enhancing the carbon catalytic activity in electron-transfer reactions, while the pyridinic nitrogens $(=\mathrm{N})$ can provide a lone pair for conjugation with the $\pi$-conjugated rings. Although amines bonded to the carbon surface are not expected to affect the electron-donor properties of carbon materials, ${ }^{12}$ it is well-known to phthalocyanine researchers and electrochemists that electropolymers of the metallo-tetra-aminophthalocyanine (MTAPc) complexes, notably NiTAPc, can easily be formed on any carbon electrode surface (for electrocalysis and sensing) using cyclic voltammetry in an appropriate organic solvent (such as DMF) in the presence of a supporting electrolyte salt (e.g., tetrabutyl ammonium phosphate, TBAP). The formation of such MTAPc electropolymers are known to be due to the electroactivity of these peripherally substituted amino groups. ${ }^{6,25}$ Furthermore, protonation of the basic pendant amine functional groups within the NiTAPc framework induces additional positive charges to the complex as well as the $\mathrm{Ni}$ (II)/Ni(III) metal centre.

Fig. 5 shows the impact of varying scan rates on the voltammetric current response at the MWCNT-NiTAPc. At higher scan rates, the observed peak-to-peak separation $\left(\Delta E_{\mathrm{p}}\right)$ increases slowly ( $c a .100 \mathrm{mV}$ ) and the ratio of anodic and cathodic peak current is almost unity, indicative of relatively fast, electrochemically quasi-reversible electron transfer kinetics.
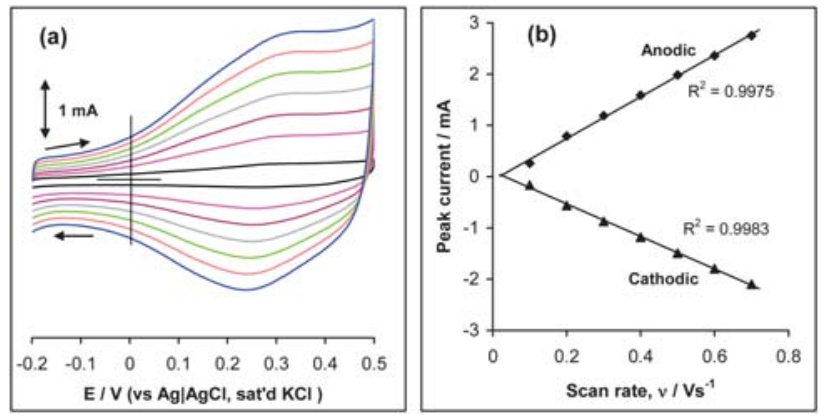

Fig. 5 (a) Typical cyclic voltammetric evolution of BPPGE- MWCNTNiTAPc at varying scan rates 100, 200, 300, 400, 500, 600 and $700 \mathrm{mVs}^{-1}$ (inner to outer). (b) Plot of peak current (anodic and cathodic) vs. scan rate. Electrolyte $=1 \mathrm{M} \mathrm{H}_{2} \mathrm{SO}_{4}$. 
A plot of peak (anodic and cathodic) current vs. scan rate was linear (Fig. 5b), indicative of a surface-bound redox system. Note that despite that we did not employ any binding substance in the fabrication process, the thin film of the MWCNT-NiTAPc nanocomposite on the BPPGE surface still exhibited excellent electrochemical stability, possibly due to the ability of the BPPGE to hold MWCNT-NiTAPc species via $\pi-\pi$ interactions.

\subsection{Galvanostatic charge/discharge characterisation}

It is well documented that galvanostatic discharge is the most reliable and accurate method for evaluating the supercapacitance of electrodes compared to either cyclic voltammetry or impedimetric methods. ${ }^{26-28}$ Fig. 6 shows typical galvanostatic charge/ discharge measurements obtained for the (i) BPPGE-MWCNT and (ii) BPPGE-MWCNT-NiTAPc at $\sim 1 \mathrm{mg} \mathrm{cm}^{-2}$ and current density of $1 \mathrm{~A} \mathrm{~g}^{-1}$; first charged to $0.5 \mathrm{~V}$ and then discharged to $-0.2 \mathrm{~V}$. The same trend was obtained at other mass loadings used (see another example obtained at $40 \mu \mathrm{g}$, Fig. 2 in the ESI†).

The specific capacitance (SC), specific power density (SP) and specific energy (SE) based on the active materials (MWCNT, or NiTAPc or MWCNT-NiTAPc) were easily estimated from the discharge process using the established eqn (3)-(5). ${ }^{26,27,29,30}$

$$
\begin{gathered}
\mathrm{SC}\left(\mathrm{F} \mathrm{g}^{-1}\right)=\frac{I \times \Delta t}{\Delta E \times m} \\
\mathrm{SP}\left(\mathrm{W} \mathrm{kg}^{-1}\right)=\frac{I \times \Delta E}{m} \\
\mathrm{SE}\left(\mathrm{W} \mathrm{h} \mathrm{Kg}{ }^{-1}\right)=\frac{I \times t \times \Delta E}{m}
\end{gathered}
$$

where $I, \Delta t, \Delta E$ and $m$ represent the current density, discharge time, potential range and the active mass of the material, respectively. The values of the $\mathrm{SC}$ at $\sim 40 \mu \mathrm{g}$ were approximately $198 \pm 11 \mathrm{~F} \mathrm{~g}^{-1}\left(40 \pm 3 \mathrm{mF} \mathrm{cm}{ }^{-2}\right)$ for the NiTAPc, $335 \pm 18 \mathrm{~F} \mathrm{~g}^{-1}$ $\left(68 \pm 4 \mathrm{mF} \mathrm{cm}^{-2}\right)$ for the MWCNT, and $981 \pm 57 \mathrm{~F} \mathrm{~g}^{-1}(200 \pm 12$ $\mathrm{mF} \mathrm{cm}{ }^{-2}$ ) for the MWCNT-NiTAPc. It is well known that in an aqueous electrolyte, capacitance is predominantly determined by the hydrophilicity of the electrode materials, the stronger the affinity of the electrode materials; the higher the double layer

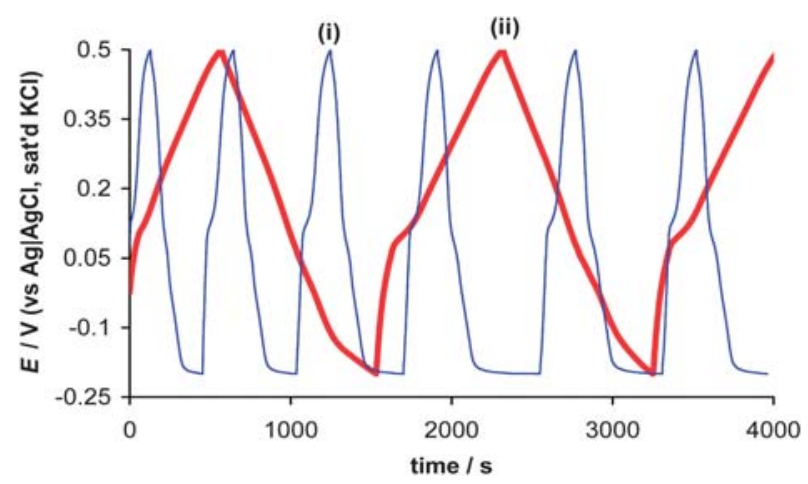

Fig. 6 Typical charge-discharge cycles obtained at BPPGE-MWCNT (i) and BPPGE-MWCNT-NiTAPc (ii) at $1 \mathrm{~A} \mathrm{~g}^{-1}$. Supporting electrolyte $=1 \mathrm{M} \mathrm{H}_{2} \mathrm{SO}_{4}$. capacitance. ${ }^{31}$ The high specific capacitance recorded for the MWCNTs should perhaps not be surprising considering the stringent acid-treaments procedure adopted in this work to functionalise our MWCNTs. For example, by merely stirring MWCNTs in 3:1 mixture of $\mathrm{H}_{2} \mathrm{SO}_{4}-\mathrm{HNO}_{3}$ for just an hour, Kim et al. ${ }^{31}$ reported the capacitance of MWCNTs immobilized onto a Ni mesh using PVDF solution in $N$-methylpyrolidine as binder (and a 3-electrode system) to be $51.3 \mathrm{~F} \mathrm{~g}^{-1}$ in $1 \mathrm{M} \mathrm{H}_{2} \mathrm{SO}_{4}$. On the other hand, Frackowiak and co-workers ${ }^{3}$ did not employ the long functionalisation times as used in our case, however, they reported values from 70 to $120 \mathrm{~F} \mathrm{~g}^{-1}$ in $6 \mathrm{M} \mathrm{KOH}$ for a 2-electrode system using a PVDF binder. Therefore, if we consider that we did not employ organic binders (which, to a certain extent, can impact negatively on the flow of ions and hence on the observed capacitance) and that the capacitance value of a 2-electrode system is about 4 times less than for a 3 -electrode system, ${ }^{1}$ the values reported by these authors ${ }^{31}$ are in close proximity to ours.

Further, we employed the galvanostatic discharge method to obtain an insight into the impact of different mass loadings (20$80 \mu \mathrm{g}$ range) on the capacitance. As seen from Fig. 7, the specific capacitance decreases slightly from the lowest mass loading $\left(\sim 1320 \mathrm{~F} \mathrm{~g}^{-1}\right.$ at $\left.20 \mu \mathrm{g}\right)$ to the highest mass $\left(\sim 907 \mathrm{~F} \mathrm{~g}^{-1}\right.$ at $\left.80 \mu \mathrm{g}\right)$.

It is interesting to observe from Fig. 7 that, within the limits of experimental errors, the SC of the MWCNT-NiTAPc nanocomposite is approximately $1000 \mathrm{~F} \mathrm{~g}^{-1}$ at all mass loadings studied. The decrease in the SC at higher mass loadings ( $>20 \mu \mathrm{g}$ ) may be attributed to the thickness of the film on the small geometric area electrode $\left(0.0196 \mathrm{~cm}^{2}\right)$ employed in this study. In fact, with the exception of the $20 \mu \mathrm{g}$ loading, all subsequent loadings had to be carried out at several "drop-dry times" to get all the materials loaded on the electrode. A thick film is disadvantageous to pseudocapacitive behaviour. As the material loading increases, more of the electroactive species are buried within the thick film and become 'redox-silent'. In addition, there will be problems with distribution of potentials within this thick surface-confined modified CNT material. Thus, as a compromise, we used $40 \mu \mathrm{g}\left(\sim 2 \mathrm{mg} \mathrm{cm}^{-2}\right)$ to compare the $\mathrm{SC}$ values of

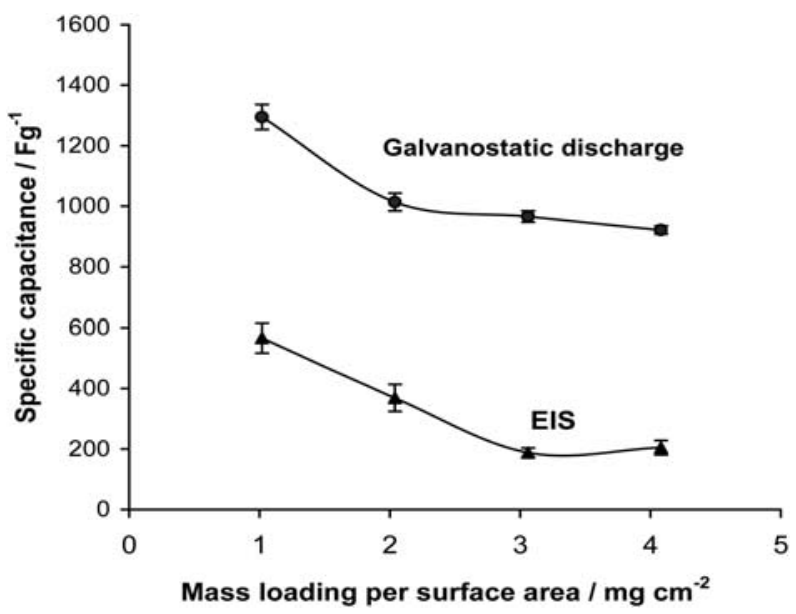

Fig. 7 Plot of specific capacitance versus mass loading of the MWCNTNiTAPc determined from the (a) galvanostatic discharge at $1 \mathrm{~A} \mathrm{~g}^{-1}$, and (b) electrochemical impedance spectroscopy at $0.3 \mathrm{~V} v s . \mathrm{Ag} \mid \mathrm{AgCl}$ (saturated $\mathrm{KCl}$ ). Supporting electrolyte $=1 \mathrm{M} \mathrm{H}_{2} \mathrm{SO}_{4}$. 
Table 1 Supercapacitive properties of basal plane pyrolytic graphite electrode (BPPGE) modified with NiTAPc, acid-treated MWCNTs and MWCNT-NiTAPc nanocomposites determined using the galvanostatic discharge method ( $n=6,95 \%$ confidence level) at $1 \mathrm{~A} \mathrm{~g}^{-1}$ (i.e., $0.1 \mathrm{~mA}$ $\mathrm{cm}^{-2}$ ) using mass loading per electrode area of $\sim 2 \mathrm{mg} \mathrm{cm}^{-2}$

\begin{tabular}{lllll}
\hline & \multicolumn{3}{l}{ Supercapacitive parameters $^{a}$} & \\
\cline { 2 - 5 } Electrode & $\mathrm{SC} / \mathrm{F} \mathrm{g} \mathrm{g}^{-1}$ & $\mathrm{SC} / \mathrm{mF} \mathrm{cm}^{-2}$ & $\mathrm{SP} / \mathrm{W} \mathrm{kg}^{-1}$ & $\begin{array}{l}\mathrm{SE} / \mathrm{W} \\
\mathrm{h} \mathrm{kg}^{-1}\end{array}$ \\
\hline BPPGE-NiTAPc & $198 \pm 11$ & $40 \pm 3$ & $703 \pm 1$ & $27 \pm 2$ \\
BPPGE-MWCNT & $335 \pm 18$ & $68 \pm 4$ & $700 \pm 1$ & $46 \pm 3$ \\
BPPGE-MWCNT- & $981 \pm 57$ & $200 \pm 12$ & $701 \pm 1$ & $134 \pm 8$ \\
NiTAPc & & & &
\end{tabular}

${ }^{a}$ Specific capacitance $\left(\mathrm{F} \mathrm{cm}^{-2}\right)$ values were calculated per geometric area of the electrode, not the electroactive area.

the three materials. The maximum supercapacitive parameters were obtained at $\sim 2 \mathrm{mg} \mathrm{cm}^{-2}$ at $1 \mathrm{~A} \mathrm{~g}^{-1}$ and are summarised in Table 1 . For the MWCNT-NiTAPc $(n=6,95 \%$ confidence level $)$ the following were obtained: $\mathrm{SC}=981 \pm 57 \mathrm{~F} \mathrm{~g}^{-1}$ or $200 \pm 12 \mathrm{mF}$ $\mathrm{cm}^{-2} ; \mathrm{SP}=701 \pm 1 \mathrm{~W} \mathrm{~kg}^{-1}$ and $\mathrm{SE}=134 \pm 8 \mathrm{~W} \mathrm{~h} \mathrm{~kg}^{-1}$. These values are more than twice higher than those of the MWCNTs alone. The values recorded with the galvanostatic discharging are more reliable. In general, the SC value for the BPPGEMWCNT-NiTAPc is indeed greater than most other half-cell or three-electrode counterparts reported in the literature. ${ }^{12,32,33}$ For example, using the same experimental conditions as ours (i.e., $1 \mathrm{~A} \mathrm{~g}^{-1}, 1 \mathrm{M} \mathrm{H}_{2} \mathrm{SO}_{4}$ with a 3-electrode system), HuliccovaJurcakova et al. ${ }^{12}$ recently reported a value of $1117 \mu \mathrm{F} \mathrm{cm}{ }^{-2}$ for the nitrogen-enriched carbon electrode. It is interesting to note that our supercapacitance value of $200 \mathrm{mF} \mathrm{cm}^{-2}$ is two orders of magnitude or more than a hundred-fold greater than the value described as 'extraordinary supercapacitance'. ${ }^{12}$ The excellent supercapacitive behaviour of the MWCNT-NiTAPc may be related to the high surface area and porous nature of the nanocomposite that enhance the penetration of solution ions. In addition, the incorporation of pendant amine groups, capable of protonation at this low $\mathrm{pH}$ incorporates more positive charges into the NiTAPc macrocycle structure than simply the Ni(II)/ $\mathrm{Ni}(\mathrm{III})$ metal centre, thus enhancing the interaction with electrolyte anions at the electrolyte-electrode interface and increasing the observed double-layer capacitance compared to macrocycles without such additional charged groups. Importantly, the thin film nature of the hybrid (small active mass, $\leq 2$ $\mathrm{mg} \mathrm{cm}{ }^{-2}$ ) shortens the distance which ions and electrons would have to travel to reach the electrode.

Also, at all current densities of $1-30 \mathrm{~A} \mathrm{~g}^{-1}$ (i.e., $0.1-3 \mathrm{mF} \mathrm{cm}^{-2}$ ) studied, the charge processes of the MWCNT-NiTAPc showed mirror images of their corresponding discharging counterparts, with no detectable IR drop even at much higher discharge current densities (see for example at 0.5 and $3 \mathrm{~mA} \mathrm{~cm}^{-2}$ Fig. 8), suggesting a very small equivalent series resistance (ESR) of the electrode. The energy deliverable efficiency $(\eta / \%)$ was obtained from eqn (6). ${ }^{34}$

$$
\eta(\%)=\frac{t_{\mathrm{d}}}{t_{\mathrm{c}}} \times 100
$$

where $t_{\mathrm{d}}$ and $t_{\mathrm{c}}$ are discharge time and charging time, respectively. The energy deliverable efficiency of the MWCNT-NiTAPc


Fig. 8 Comparative charge-discharge curves at $5 \mathrm{Ag}^{-1}$ (a) and $30 \mathrm{Ag}^{-1}$ (b) obtained with the BPPGE-MWCNT-NiTAPc composite. Supporting electrolyte $=1 \mathrm{M} \mathrm{H}_{2} \mathrm{SO}_{4}$.

nanocomposite at the $\sim 2 \mathrm{mg} \mathrm{cm}^{-2}$ was $\sim 121 \%$ at $1 \mathrm{~A} \mathrm{~g}^{-1}$ and up to $\sim 100 \%$ at $10 \mathrm{~A} \mathrm{~g}^{-1}$. To obtain an insight into the impact of phthalocyanine ring substituents on the supercapacitive behaviour, similar galvanostatic charge/discharge experiments for NiTAPc-MWCNT were also performed with unsubstituted nickel phthalocyanine (NiPc) and nickel(II) tetra-tert-butyl phthalocyanine $(t \mathrm{BuNiPc})$. Interestingly, the NiTAPc gave superior capacitive data. As an example, at a current density of $1 \mathrm{Ag}^{-1}$ (for $\sim 20 \mu \mathrm{g}$ loading) the specific capacitance decreases as NiTAPc $\left(\sim 243 \mathrm{~F} \mathrm{~g}^{-1}\right)>\operatorname{NiPc}\left(\sim 176 \mathrm{~F} \mathrm{~g}^{-1}\right)>t$ BuNiPc $\left(\sim 126 \mathrm{~F} \mathrm{~g}^{-1}\right)$.

This trend is also evident from their comparative CV evolutions (Fig. 3 in the ESI $\dagger$ ). This trend is in accordance with the nitrogen content of the three phthalocyanine species. In addition, the poorer value of the $t \mathrm{BuNiPc}$ may be related to the absence of nitrogens with any donor lone pairs to protonate at this acidic $\mathrm{pH}$ used in this work and/or the bulky tert-butyl group that may deform the phthalocyanine framework, thus reducing the supercapacitance. In addition, Kim et al. ${ }^{31}$ proved that alkyl chains reduce supercapacitance. In addition, the NiTAPcMWCNT is more stable on the BPPGE and highly reproducible galvanostatic data than the other nickel phthalocyanines studied. More importantly, NiPc and $t \mathrm{BuNiPc}$ and their MWCNT composites are less soluble in DMF than the NiTAPc based electrodes. This superior performance of the NiTAPc is not fully understood at this moment, but may be related to its nitrogencontaining groups, which have recently been observed to greatly enhance supercapacitive behaviour. ${ }^{10-12}$

The stability of the BPPGE-MWCNT-NiTAPc was tested by continuous cycling at high current density $\left(1 \mathrm{~mA} \mathrm{~cm}^{-2}\right)$. A typical repetitive charge-discharge cycling for 1500 cycles, lasting about 3 days, is shown in Fig. 9. The Figure clearly shows that the electrode is able to charge and discharge continuously without any significant loss in capacitance.

\subsection{Impedance spectroscopy characterisation}

Unlike the galvanostatic discharge method, electrochemical impedance spectroscopy (EIS) is the least reliable and accurate technique for determining the supercapacitive properties of materials, especially those related to conducting polymeric 


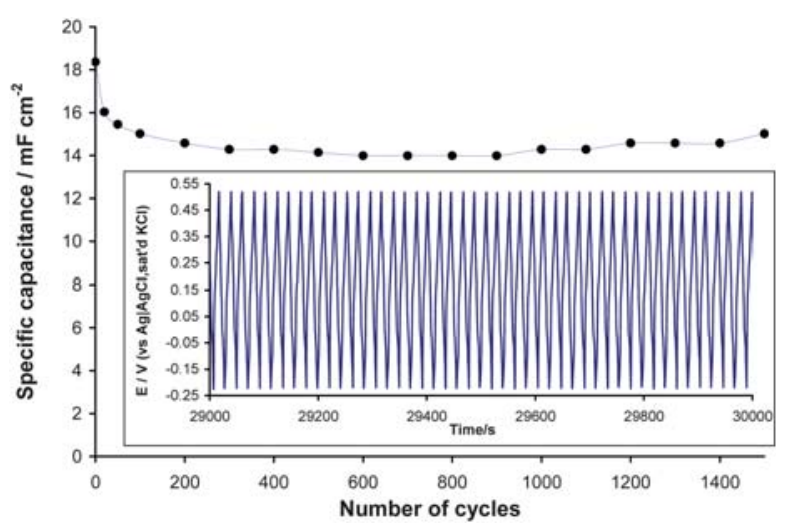

Fig. 9 Variation of specific capacitance with the number of cycles at 1 $\mathrm{mA} \mathrm{cm}{ }^{-2}$. Inset is a portion of the charge-discharge curves obtained during the 3-day repetitive cycling. Supporting electrolyte $=1 \mathrm{M} \mathrm{H}_{2} \mathrm{SO}_{4}$.

substances. ${ }^{35-40}$ Fig. 10 shows typical comparative Nyquist plots of the electrodes obtained at $20 \mu \mathrm{g}$ material loading $\left(\sim 1 \mathrm{mg} \mathrm{cm}^{-2}\right)$.

The low-frequency differential capacitance $\left(C_{\mathrm{d}}\right)$ for each of the electrodes can be obtained from the slope $\left(1 / 2 \pi C_{\mathrm{d}}\right)$ of the plot of the imaginary component of the impedance versus the reciprocal of the frequency (i.e., $\left.-Z^{\prime \prime} v s .1 / f\right){ }^{29,40,41}$ The values of the specific capacitances for the three modifiers at $\sim 2 \mathrm{mg} \mathrm{cm}^{-2}$ fixed at $0.3 \mathrm{~V}$ were approximately $13 \mathrm{~F} \mathrm{~g}^{-1}$ for the NiTAPc, $64 \mathrm{~F} \mathrm{~g}^{-1}$ for the MWCNT and $340 \mathrm{~F} \mathrm{~g}^{-1}$ for the MWCNT-NiTAPc nanocomposite. These SC values are indicative of strong deviation from the data obtained from the galvanostatic method. This strong discrepancy has also been observed by several workers for conducting polymeric substances, ${ }^{35-40}$ and the origin of which has long been a subject of some controversy for more than two decades. For example, Murray and co-workers ${ }^{35}$ have suggested the involvement of some physico-chemical heterogeneities; Tanguy and co-workers ${ }^{37}$ attributed such phenomenon to "deeply trapped" counter ions which remain immobile during impedance experiment; while Kalaji and Peter ${ }^{39}$ described it as the consequence of slow conformational changes occurring in the polymer network. Later, Ren and Pickup ${ }^{40}$ performed further

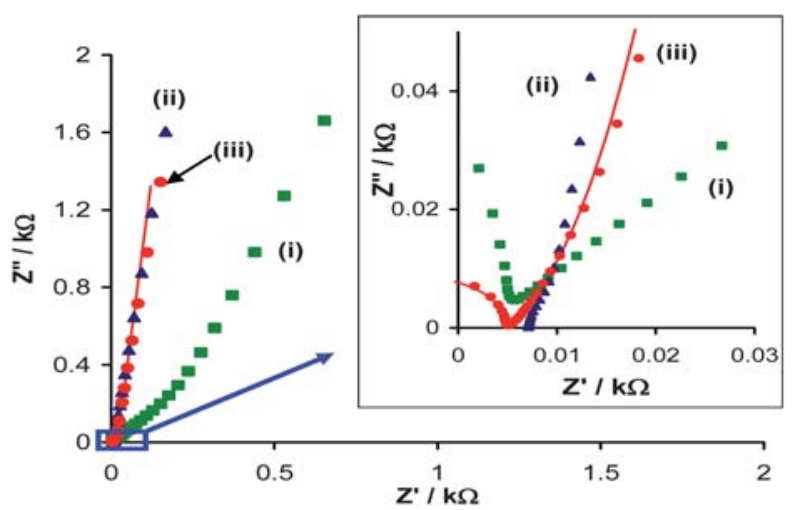

Fig. 10 Comparative Nyquist plots of BPPGE-NiTAPc (i), BPPGEMWCNT (ii) and BPPGE-MWCNT-NiTAPc (iii) in $1 \mathrm{M} \mathrm{H}_{2} \mathrm{SO}_{4}$ at $0.30 \mathrm{~V}$, between $100 \mathrm{kHz}$ and $10 \mathrm{mHz}$. Inset: The expanded portion of the high frequency region, showing the fitted curve for the BPPGEMWCNT-NiTAPc. experiments that claimed to support the arguments on conformational changes. It should be noted that recent workers have also observed this discrepancy and attributed it to "redoxswitching hysteresis" associated with conducting polymers, ${ }^{42}$ or the hindrance that accompanies the penetration of alternating current into the bulk electrode. ${ }^{27}$ As also observed in Fig. 7, the $\mathrm{SC}$ value of the different mass loading of the MWCNT-NiTAPc decreases significantly from the lowest mass loading $\left(\sim 586 \mathrm{~F} \mathrm{~g} \mathrm{~g}^{-1}\right.$ at $20 \mu \mathrm{g})$ to the highest mass $\left(\sim 180 \mathrm{~F} \mathrm{~g}^{-1}\right.$ at $\left.80 \mu \mathrm{g}\right)$. Thus, it is very likely that one or more of these interpretations by other workers may also hold in our case, especially considering that thick films are prone to inhibiting facile current flow and exacerbating the trapping and/or immobility of ions. Since this behaviour has mainly been reported for the conducting polymers, it suggests that the MWCNT-NiTAPc nanocomposites exhibit some properties of conducting polymeric substances. Also, it should be noted from Fig. 6 that the SC values obtained from the EIS and galvanostatic discharge techniques are in the same magnitude. This is interesting considering that other workers ${ }^{43}$ have observed that the value of SC obtained from galvanostatic is about a magnitude greater than the value obtained from EIS experiment.

Despite the paucity of EIS in establishing reliable data on the supercapacitive data, it still remains a powerful technique for providing valuable insights into processes that occur at the electrode/solution interface. As seen in Fig. 10, the BPPGEMWCNT shows near-vertical line as expected for pseudocapacitor. The BPPGE-MWCNT-NiTAPc (inset) shows smaller incomplete semi-circle in the high frequency region (describing the ESR or charge transfer resistance across the electrode/electrolyte interface), compared to the BPPGE-NiTAPc, followed by a near-vertical line at low frequency region, which is typical of capacitive behaviour. The transition point between the high frequency and low frequency component, referred to as the "knee" or "onset" frequency $\left(f_{\mathrm{o}}\right)$ describes the maximum frequency at which the capacitive behaviour is dominant, and is a measure of the power capability of a supercapacitor; the higher the $f_{\mathrm{o}}$ the more rapidly the supercapacitor can be charged and discharged or the higher the power density that can be achieved from the supercapacitor. ${ }^{44,45}$ The values of the $f_{\mathrm{o}}$ decreases as MWCNT-NiTAPc $(720 \mathrm{~Hz}) \gg$ NiTAPc $\sim \operatorname{MWCNT}(\sim 4 \mathrm{~Hz})$. Accordingly, the higher $f_{\mathrm{o}}$ value of the MWCNT-NiTAPc confirms its higher power property compared to the individual NiTAPc and MWCNTs, which is consistent with the comparative CV data shown in Fig. 4. The reciprocal of the $f_{\mathrm{o}}$ is the response time of the capacitor. Note that the $f_{\mathrm{o}}$ of MWCNTNiTAPc is about $1.4 \mathrm{~ms}$, suggesting that most of its stored energy is still accessible at frequencies as high as $720 \mathrm{~Hz}$. Most commercially available supercapacitors, including those specifically designed for higher power applications, operate at frequencies less than $1 \mathrm{~Hz}^{46}$

The impedimetric behaviour of MWCNT-NiTAPc could be satisfactorily modelled by the electrical equivalent circuit comprising the modified Randles circuit with one Voigt RC element (Fig. 11), involving a solution resistance $\left(R_{\mathrm{s}}=-11.7 \Omega\right)$, a double-layer capacitance $\left(C_{\mathrm{d} 1}=95 \mathrm{nF}\right)$, an electron-transfer resistance $\left(R_{\mathrm{ct} 1}=16.6 \Omega\right.$ and $\left.R_{\mathrm{ct} 2}=18 \Omega\right)$ and constant-phase elements $\left(\mathrm{CPE}_{1}=10.5 \mathrm{mF}\right.$ with $n_{1}=0.95$ and $\mathrm{CPE}_{2}=33.6 \mathrm{mF}$ with $\left.n_{2}=0.55\right)$. 


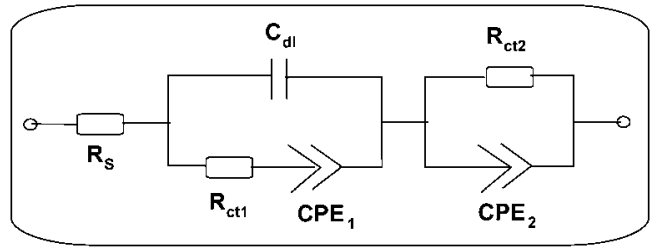

Fig. 11 Electrical equivalent circuit used in fitting the experimental EIS data obtained at the BPPGE-MWCNT-NiTAPc.

Note that the impedance of CPE is defined as in eqn (7): ${ }^{47,48}$

$$
Z_{\mathrm{CPE}}=\frac{1}{\left[Q(j \omega)^{n}\right]}
$$

where $Q$ is the frequency-independent constant relating to the surface electroactive properties, $\omega$ is the radial frequency, the exponent $n$ arises from the slope of $\log Z v s$. $\log f$ (and has values $-1 \leq n \leq 1)$. If $n=0$, the CPE behaves as a pure resistor; $n=1$, CPE behaves as a pure capacitor, $n=-1$ CPE behaves as an inductor; while $n=0.5$ corresponds to Warburg impedance $\left(Z_{\mathrm{w}}\right)$ which is associated with the domain of mass transport control arising from the diffusion of ions to and from the electrode|solution interface. The $\mathrm{CPE}_{1}$ that replaced the diffusion impedance $\left(Z_{\mathrm{w}}\right)$ in the ideal Randles circuit is associated with a high $n_{1}$ value $(0.95)$, describing the high porous nature of the electrode. The $n_{2}$ value $(0.55)$ is approximately equal to the ideal Warburg behaviour of $n=0.5$, describing the diffusion of ions through the thin film. ${ }^{29}$ From the Bode plot $(\log |Z| v s . \log f$ (Fig. 12), the high frequency region yielded a slope of close to zero, characteristic of a pure resistor, while the low frequency region yielded a slope of $<1.0$, characteristic of a pseudocapacitor. Note that the same trend of impedimetric evolutions observed in Fig. 10 was also observed at higher mass loading (see example for $2 \mathrm{mg} \mathrm{cm}^{-2}$, Figure SI 4), with the MWCNT-NiTAPc nanocomposite still giving the highest $f_{\mathrm{o}}$ of $730 \mathrm{~Hz}$.

In general, these data clearly suggest that the impedance of this electrode varies from a pure resistor at high frequency to pseudocapacitor at low frequency region. Surprisingly, all attempts to replace the ideal $\mathrm{C}_{\mathrm{dl}}$ with a $\mathrm{CPE}$ (a real application situation) in the modeling circuit proved unsuccessful. As has elegantly been described recently by Orazem and Tribollet, ${ }^{45}$ frequency dispersion leading to CPE behaviour occurs as a result of distribution

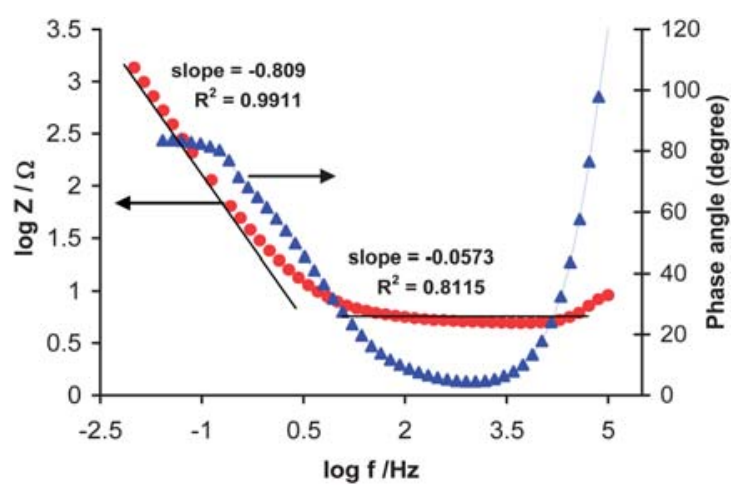

Fig. 12 Typical Bode plots for the BPPGE-MWNCT-NiTAPc electrode. of time constants along either the area of the electrode surface (involving a 2-dimensional aspect of the electrode) or along the axis normal to the electrode surface (involving a 3-dimensional surface). It is noteworthy that a 2-D distribution presents itself as an ideal $\mathrm{RC}$ behaviour, meaning that impedance measurements are very useful in distinguishing whether the observed global $\mathrm{CPE}$ behaviour is due to a 2-D or 3-D distribution or both.

Thus, we may conclude that the observed impedimetric behaviour of the BPPGE-MWCNT-NiTAPc likely involve time constant distributions occuring along the area of the electrode as well as along the axis normal to the electrode. Also, from the other type of Bode plot (i.e., - phase angle $(\theta) v s . \log f$, (Fig. 12), the $\theta$ was about $80^{\circ}$, which is less than the $90^{\circ}$ expected of an ideal capacitive behaviour, thus further confirming the presence of CPE and pseudocapacitive nature of the BPPGEMWCNT-NiTAPc.

\section{Conclusion}

In conclusion, we have proved for the first time that an CNT-MPc modified electrode shows excellent supercapacitive behaviour. Given the high number of existing MPc complexes and new ones constantly reported in the literature, and the importance of supercapacitors in energy development, we envisage that this novel study is likely to spark research interests in the supercapacitive properties of CNT-MPc hybrid electrodes.

\section{Acknowledgements}

We thank NRF (GUN \# 2073666, 65305, 68338) and CSIR for their financial support. A. T. C. thanks NRF for an MSc bursary, B. O. A. thanks NRF for a scarce skill postdoctoral fellowship.

\section{References}

1 R. Kotz and M. Carlen, Electrochim. Acta, 2000, 45, 2483.

2 X. Liu and P. G. Pickup, Energy Environ. Sci., 2008, 1, 494.

3 E. Frackowiak and F. Béguin, Carbon, 2001, 39, 937.

4 G. de la Torre, C. G. Claessens and T. Torres, Chem. Commun., 2007, 2000.

5 The Porphyrin Handbook, vol. 15-20, ed. K. M. Kadish, K. M. Smith and R. Guilard, Academic Press, Boston, 2003, (ch. 97-122).

6 K. I. Ozoemena, T. Nyokongin Encyclopedia of Sensors, vol. 3, ed. C. A. Grimes, E. C. Dickey and M. V. Pishko, American Scientific Publishers, California, 2006, ch. E, pp. 157-200.

7 J. H. Zagal, S. Griveau, K. I. Ozoemena, T. Nyokong and F. Bedioui, J. Nanosci. Nanotechnol., 2009, 9, 2201.

$8 \mathrm{H}$. Take, H. Nonoue, Electric double layer capacitor and method for manufacturing electric double layer capacitor, U.S. Pat. Appl. Publ., 2007, US 2007/206343 A1, 10 pp.

9 Y. Zhang, H. Feng, X. Wu, L. Wang, A. Zhang, T. Xia, H. Dong, X. Li and L. Zhang, Int. J. Hydrogen Energy, 2009, 34, 4889.

10 E. Frackowiak, G. Lota, J. Machnikowski, C. Vix-Guterl and F. Béguin, Electrochim. Acta, 2006, 51, 2209.

11 D. Hulicova, M. Kodama and H. Hatori, Chem. Mater., 2006, 18, 2318.

12 D. Hulicova-Jurcakova, M. Kodama, S. Shiraishi, H. Hatori, Z. H. Zhu and G. Q. Lu, Adv. Funct. Mater., 2009, 19, 1.

13 V. Gupta and N. Miura, J. Power Sources, 2006, 157, 616.

14 J. Liu, A. G. Rinzler, H. Dai, J. H. Hanfer, R. K. Bradley, P. J. Boul, A. Lu, T. Iverson, K. Shelimov, C. B. Huffman, F. R. Macias, Y. S. Shon, T. R. Lee, D. T. Colbert and R.E., Science, 1998, 280, 1253.

15 Z.-L. Yang, H.-Z. Chen, L. Cao, H.-Y. Li and M. Wang, Mater. Sci. Eng., B, 2004, 106, 73. 
16 M. Siswana, K. I. Ozoemena and T. Nyokong, Electrochim. Acta, 2006, 52, 114.

17 J. Pillay and K. I. Ozoemena, Electrochim. Acta, 2007, 52, 3630.

18 B. N. Acher and P. K. Jayasree, Synth. Met., 1999, 104, 101.

19 K. I. Ozoemena and T. Nyokong, Inorg. Chem. Commun., 2003, 6, 1192.

20 X. Wang, Y. Liu, W. Qiu and D. Zhu, J. Mater. Chem., 2002, 12, 1636.

21 H. Murakami, T. Nomura and N. Nakashima, Chem. Phys. Lett., 2003, 378, 481-485.

22 C. A. Thorogood, G. G. Wildgoose, A. Crossley, R. M. J. Jacobs, J. H. Jones and R. G. Compton, Chem. Mater., 2007, 19, 4964-4974.

23 C. A. Thorogood, G. G. Wildgoose, J. H. Jones and R. G. Compton, New J. Chem., 2007, 31, 958-965.

24 G. G. Wildgoose, P. Abiman and R. G. Compton, J. Mater. Chem., 2009, 19, 4875-4886.

25 F. Bedioui, S. Griveau, T. Nyokong, A. J. Appleby, C. A. Caro, G. M. Gulppi Ochoa and J. H. Zagal, Phys. Chem. Chem. Phys., 2007, 9, 3383.

26 K. R. Prasad and N. Munichandraiah, Electrochem. Solid-State Lett., 2002, 5, A271.

27 V. Gupta, T. Shinomiya, N. Miurain: Recent Advances in Supercapacitors, ed. V. Gupta, Transworld Research Network, Kerala, India, 2006, (ch. 2), pp. 17-28.

28 K. Kierzek, E. Frackowiak, G. Lota, G. Gryglewicz and J. Machnikowski, Electrochim. Acta, 2004, 49, 515.

29 V. Ganesh, S. Pitchumani and V. Lakshminarayanan, J. Power Sources, 2006, 158, 1523.

30 T. Shinomiya, V. Gupta and N. Miura, Electrochim. Acta, 2006, 51, 4412.

31 Y.-T. Kim, Y. Ito, K. Tadai and T. Mitani, Appl. Phys. Lett., 2005, 87, 234106.
32 H. Zhang, G. cao, Z. Wang, Y. Yang, Z. Shi and Z. Gu, Electrochem. Commun., 2008, 10, 1056.

33 V. Gupta, S. Gupta and N. Miura, J. Power Sources, 2008, 177, 685.

34 S. G. Kandalkar, J. 1. Gunjakar and C. D. Lokhande, Appl. Surf. Sci., 2008, 254, 5540 .

35 B. J. Feldman, P. Burgmayer and R. W. Murray, J. Am. Chem. Soc., $1985, \mathbf{1 0 7}, 872$.

36 N. Mermilliod, J. Tanguy and F. Petior, J. Electrochem. Soc., 1986, 133, 1073.

37 J. Tanguy, N. Mermilliuod and M. Hoclet, J. Electrochem. Soc., 1987, 134, 795.

38 T. Tanguy, M. Slama, M. Hoclet and J. L. Baudin, Synth. Met., 1989, 28, 145.

39 M. Kalaji and L. M. Peter, J. Chem. Soc., Faraday Trans., 1991, 87, 853.

40 X. Ren and P. G. Pickup, J. Electroanal. Chem., 1994, 372, 289.

41 T. C. Girija and M. V. Sangaranarayanan, J. Appl. Electrochem., 2006, 36, 531.

42 G. Xu, W. Wang, X. Qu, Y. Yin, L. Chu, B. He, H. Wu, J. Fang, Y. Bao and L. Liang, Eur. Polym. J., 2009, DOI: 10.1016/ j.europolymj.2009.05.016.

43 W. Sun and X. Chen, J. Power Sources, 2009, 193, 924.

44 C. Du and N. Pan, J. Power Sources, 2006, 160, 1487.

45 C.-W. Huang, Y.-T. Wu, C.-C. Hu and Y.-Y. Li, J. Power Sources, 2007, 172, 460.

46 J. R. Miller, Proceedings of the Electrochemical Society Meeting, Chicago, October, 1995, 246.

47 M. E. Orazem, B. Tribollet, Electrochemical Impedance Spectroscopy, John Wiley \& Sons Inc, Hoboken, NJ., 2008, (ch. 13).

48 E. Barsoukov, J. R. Macdonald, Impedance Spectroscopy: Theory Experiment, and Applications, 2nd edn, Wiley, Hoboken, New Jersey, 2005, (ch. 2), p. 37 